Kansas State University Libraries

New Prairie Press

\title{
SELECTION OF BLOCKED TWO-LEVEL FRACTIONAL FACTORIAL DESIGNS FOR AGRICULTURAL EXPERIMENTS
}

Weiming Ke

Cuirong Ren

Huitian Lu

Follow this and additional works at: https://newprairiepress.org/agstatconference

Part of the Agriculture Commons, and the Applied Statistics Commons

\section{(c) (1) $\Theta$}

This work is licensed under a Creative Commons Attribution-Noncommercial-No Derivative Works 4.0 License.

\section{Recommended Citation}

Ke, Weiming; Ren, Cuirong; and Lu, Huitian (2007). "SELECTION OF BLOCKED TWO-LEVEL FRACTIONAL FACTORIAL DESIGNS FOR AGRICULTURAL EXPERIMENTS," Conference on Applied Statistics in Agriculture. https://doi.org/10.4148/2475-7772.1109

This is brought to you for free and open access by the Conferences at New Prairie Press. It has been accepted for inclusion in Conference on Applied Statistics in Agriculture by an authorized administrator of New Prairie Press. For more information, please contact cads@k-state.edu. 


\title{
SELECTION OF BLOCKED TWO-LEVEL FRACTIONAL FACTORIAL DESIGNS FOR AGRICULTURAL EXPERIMENTS
}

\author{
Weiming $\mathrm{Ke}^{1}$, Cuirong $\mathrm{Ren}^{2}$, and Huitian $\mathrm{Lu}^{3}$ \\ ${ }^{1}$ Department of Mathematics and Statistics \\ ${ }^{2}$ Department of Plant Sciences \\ ${ }^{3}$ Department of Engineering Technology \& Management \\ South Dakota State University, Brookings, SD 57006, U.S.A
}

\begin{abstract}
Blocked two-level fractional factorial designs are a very useful tool for efficient data collection in agricultural and other scientific research. In most experiments, in addition to the main effects, some two-factor interactions are also meaningful and need to be estimated. We propose a method for efficiently selecting blocked two-level fractional factorial designs when some of the two-factor interactions are non-negligible. We then present some results for a design with only 8 or 16 runs to illustrate how to use this method.
\end{abstract}

Key words and phrases: blocking factor; defining contrast subgroup; defining words; minimum aberration; resolution; word-length pattern; confounding pattern.

\section{Introduction}

Two-level fractional factorial $\left(2^{m-p}\right)$ designs allow us to study many factors with relatively small run size. They are very useful for identifying important factors and are widely used in many areas of scientific investigation. The practical and theoretical importance of this class of designs has long been established by Box, Hunter, and Hunter (1978). Since the fraction can be chosen in many different ways, a key concern is how to choose a fraction of the full factorial design for a given run size and the number of factors. Commonly used criterion for $2^{m-p}$ design selection is the minimum aberration criterion proposed by Fries and Hunter (1980). For a small number of factors, Box, Hunter, \& Hunter (1978) provided a useful catalogue of $2^{m-p}$ designs with minimum aberration. Franklin (1984) constructed more minimum aberration designs. A more complete catalogue of $2^{m-p}$ designs, ordered by the minimum aberration criterion, was provided by Chen, Sun, and $\mathrm{Wu}(1993)$.

In agricultural experiments, in order to reduce extraneous error and increase the precision of inferences, it is often desirable to group the experimental units into blocks such as different fields in a plant study. Hence blocked $2^{m-p}$ designs are often used in order to make data collection more efficient. Work on the criteria of optimal blocking schemes includes: Bisgaard (1994), Sun, Wu, 
and Chen (1997), Sitter, Chen, and Feder (1997), Chen and Cheng (1999), Cheng and Wu (2002), and Cheng, Li, and Ye (2004). Their schemes yield optimal estimation of models containing main effects and block effects.

In some agricultural experiments, in addition to the main effects and blocking effects, some 2-factor interactions may be important and should be estimated. For unblocked designs, much work has been done on constructing designs that allow estimation of a set of specified effects, including some 2-factor interactions: Addelman (1962), Greenfield (1976), Franklin (1985), Hedayat and Pesotan (1992), Wu and Chen (1992), Tang and Deng (1999), Dey and Suen (2002), Ke and Tang (2003), and Ke, Tang, and Wu (2005).

In this paper, we propose a method to select blocked $2^{m-p}$ designs for the models containing some two-factor interactions. We then discuss how to search for designs using this method and present some results for designs of 8 and 16 runs. Section 2 of the paper introduces two-level fractional factorial designs and blocking. Section 3 introduces and discusses a method for selecting blocked $2^{m-p}$ designs for models containing some non-negligible 2-factor interactions. Section 4 examines how to search for designs using this method and presents some results for designs of 8 and 16 runs. Section 5 concludes the paper with an illustrative example.

\section{Two-level fractional factorial designs and blocking}

\subsection{Two-level fractional factorial designs}

A regular two-level fractional factorial design is commonly referred to as a $2^{m-p}$ design. It has $m$ two-level factors with $2^{m-p}$ runs, and is completely determined by $p$ independent defining relations. When $p=0$, a full factorial $2^{m}$ design is produced. A defining relation is given by a word of letters which are labels of factors denoted by $1,2, \ldots, m$. The number of letters in a word is called its word-length. The group of defining words generated by the $p$ independent defining words is called the defining contrast subgroup for the design. The length of the shortest word in the defining contrast subgroup is called the resolution of a design. The vector $W(d)=\left(A_{1}(d)\right.$, $\left.A_{2}(d), \ldots, A_{m}(d)\right)$ is called the word-length pattern of the design $d$, where $A_{i}(d)$ is the number of words of length $i$ in the defining contrast subgroup. The resolution of a design is the smallest $r$ satisfying $A_{r} \geq 1$. The resolution criterion proposed by Box and Hunter (1961) selects $2^{m-p}$ designs that have highest resolution. Since two designs having the same resolution may have different word-length patterns and may not be equally good, Fries and Hunter (1980) proposed the minimum aberration criterion to further discriminate among $2^{m-p}$ designs. For two designs $d_{1}$ and $d_{2}$, suppose $r$ is the smallest value such that $A_{r}\left(d_{1}\right) \neq A_{r}\left(d_{2}\right)$. We say that $d_{1}$ has less aberration than $d_{2}$ if $A_{r}\left(d_{1}\right)<A_{r}\left(d_{2}\right)$. If no design has less aberration than $d_{1}$, then $d_{1}$ is said to have minimum aberration. The minimum aberration criterion which selects $2^{m-p}$ designs with minimum aberration is a commonly used criterion for $2^{m-p}$ design selection (Wu and Hamada, 2000).

\section{Example 2.1.1 - The $2^{4-1}$ Minimum Aberration Design:}

Suppose we wish to perform an experiment with 8 runs and several factors at two levels, labeled +1 and -1 . Table 1 gives the columns of an 8 -run saturated design with its columns 
arranged in Yates' order, with the generating independent columns 1, 2, and 4, in boldface. The Yates' order of the columns of an 8-run saturated design can be written as $\left(\boldsymbol{a}_{\mathbf{1}}, \boldsymbol{a}_{\mathbf{2}}, a_{1} a_{2}, \boldsymbol{a}_{\mathbf{3}}, a_{1} a_{3}\right.$, $a_{2} a_{3}, a_{1} a_{2} a_{3}$ ) where $\boldsymbol{a}_{\mathbf{1}}, \boldsymbol{a}_{\mathbf{2}}$, and $\boldsymbol{a}_{\mathbf{3}}$ are three independent columns. Hence columns 3,5 , 6, and 7 can be obtained by multiplying columns 1 and 2, 1 and 4, 2 and 4, and 1,2, and 4, respectively.

If we use columns 1,2 , and 4 in Table 1 to set the levels of three factors $\mathrm{A}, \mathrm{B}$, and $\mathrm{C}$, respectively, then $y_{1}$ through $y_{8}$ represent the responses at the $2^{3}=8$ possible combinations of factor settings. This gives a $2^{3}=8$ run, two-level, three-factor, full factorial design. By using this $2^{3}$ full factorial design, the main effects of $A, B$, and $C$, as well as their interactions $A B, A C, B C$, and $A B C$ can be estimated. If we would like to study one more factor $D$ using the 8-run design, we have different choices. For design $d_{1}$, we assign the levels of factor $D$ to column 7 . This gives a $2^{4-1}$ fractional factorial design. Since $7=(1)(2)(4)$ or $I=(1)(2)(4)(7)$ where $I$ denotes the column of +1 's, the estimate for the main effect $D$ could not be separated from the effect of the interaction between $A, B$, and $C$. That is $D=A B C$, or $I=A B C D$. Here $I=(1)(2)(4)(7)$ is the defining relation or defining word of the $2^{4-1}$ design. The resolution of the design is 4 and the word-length pattern $W\left(d_{1}\right)=(0,0,0,1)$. For design $d_{2}$, we assign factor $D$ to column $6=(2)(4)$. The defining word is $I=(2)(4)(6)$ and the resolution is 3 . The word-length pattern $W\left(d_{2}\right)=(0,0,1,0)$. Obviously, $d_{1}$ is better than $d_{2}$ because it has higher resolution and minimum aberration: $A_{3}\left(d_{1}\right)=0<A_{3}\left(d_{2}\right)=1$. Both definitions of resolution and minimum aberration are based on the hierarchical assumption: (i) lower order interactions are more important than higher order interactions, (ii) effects of the same order are equally important. The advantage of $d_{1}$ is obvious based on the resolution principle, because the main effects in $d_{1}$ are confounded with three-factor interactions whereas the main effects in $d_{2}$ are confounded with two-factor interactions. The 8-run $2^{m-p}$ designs can be used to study up to seven factors by assigning these factors to all the seven columns. This design is denoted by $2^{7-4}$, and called a saturated design.

\subsection{Blocking and its applications}

Blocking is a commonly used technique to control systematic variation in experiments. Such variation might occur from field-to-field, day-to-day, or batch-to batch. Without blocking, the systematic variation can influence the accuracy and efficiency of effect estimation. Blocking can effectively eliminate the systematic variance by grouping the runs of an experiment into blocks. In a blocked design, the variance due to blocks is modeled. Hence it is removed from the residual variance; thereby effectively reducing the magnitude of the estimated experimental error. Criteria for the choice of blocks are most frequently different settings or environments for the conduct of the experiment. In any case, blocks should be chosen so that the units within blocks are as homogeneous as possible. Blocking can be accomplished through the use of blocking factors in a design. For $2^{m-p}$ designs, since there are many choices to assign blocking factors to the unused columns of a saturated design, blocking schemes and methods are needed to select columns that reduce the bias (as one did in the selection of unblocked designs). In blocked $2^{m-p}$ designs, interactions between treatment and blocking factors are assumed to be non-existent, a necessary condition for the effectiveness of blocking. See Box, Hunter, \& Hunter (1978) for the principles and assumptions in the construction of block designs. For the models including only main effects and blocking effects, many blocking schemes for $2^{m-p}$ designs have been discussed in the 
literature. In this article, we discuss the blocking schemes for the models that include some important two-factor interactions in addition to the main effects and the blocking effects.

\section{A method for selecting blocked $2^{m-p}$ designs}

\subsection{The criterion for selecting blocked $2^{m-p}$ designs}

For unblocked $2^{m-p}$ designs, Ke and Tang (2003) proposed a minimum $N$-aberration criterion to select designs by systematically minimizing the bias of all effects in the model caused by other effects. This criterion was further studied and summarized by Cheng and Tang (2005). In this article, we consider blocked $2^{m-p}$ designs that allow estimation of some 2-factor interactions. Suppose we wish to estimate main effects and some important 2-factor interactions using a blocked $2^{m-p}$ design. The fitted model should include all main effects, blocking effects, and the important 2-factor interactions. If the effects not in the postulated model cannot be completely ignored (i.e., are non-negligible), they will bias the estimates of the effects in the model. To solve this problem, the key issues are to permit estimation of the main effects, blocking effects, and the important 2-factor interactions in the model and to minimize the bias caused by the other effects not included in the model. The optimal design should be selected to minimize contamination of effect estimates by any unknown non-negligible effects excluded from the model. The proposed criterion is given below:

Optimal design selection criterion: Suppose that the postulated model includes main effects, blocking effects, and some important two-factor interactions. Let $N_{j}, j=2,3, \ldots, m$, be the number of $j$-factor interactions not in the model confounded with the effects in the model. We select optimal blocked $2^{m-p}$ designs by sequentially minimizing $N_{2}, \ldots, N_{m}$.

To gain further insight into the criterion, we now examine the criterion in detail. When some 2-factor interactions need to be estimated, the postulated model should consist of all main effects, blocking effects and these important 2-factor interactions. Those 2-factor interactions not in the model and other higher-order interactions generally cause a bias on the estimation of the effects in the model. The measure of this bias, as given by $N_{\mathrm{j}}$, is the number of the $j$-factor interactions outside the model that are confounded with the effects in the model. Under the hierarchical assumption that lower-order effects are more important than higher-order effects (Wu and Hamada (2000)), to minimize the bias, we should sequentially minimizing $N_{2}, N_{3}, \ldots$, $N_{m}$. The vector $\left(N_{2}, N_{3}, \ldots, N_{m}\right)$ is called the confounding pattern of a design. Hence this criterion selects as optimal the design that has minimum $N_{2}$. If several designs have the same number of $N_{2}$, we select as optimal the design that has minimum $N_{3}$ among the designs that have minimum $N_{2}$, and so on. Note that $N_{3}$ is the total number of 3-factor interactions that are confounded with the effects in the model.

\section{Example 3.1.1 - Selecting a Blocked $2^{4-1}$ Minimum N-aberration Design}

Suppose that we want to study four factors $A, B, C$, and $D$, and two-factor interactions $A B$ and $A C$ by using a blocked design of 8 runs. We consider two designs $d_{1}$ and $d_{2}$. Using Table 1, for $d_{1}$, we assign the four treatment factors $A, B, C$, and $D$ to columns $1,4,7$, and 2 , and the 
blocking factor to column $3_{b}$, where $b$ indicates blocking factor. The interactions to be estimated should be (1)(4) and (1)(7). Since $7=(1)(2)(4)$ and $3_{b}=(1)(2)$, the defining contrast subgroup of the design is given by $I=(1)(2)\left(3_{b}\right)=\left(3_{b}\right)(4)(7)=(1)(2)(4)(7)$. Hence we have the following (model effect $=$ non-model confounded effect) identity pairs: $(1)(4)=(2)(7),(1)(7)=(2)(4), 3_{b}=$ $(1)(2), 3_{b}=(4)(7), 1=(2)(4)(7), 2=(1)(4)(7), 4=(1)(2)(7)$, and $7=(1)(2)(4)$. Therefore $N_{2}=4$, $N_{3}=4$, and $N_{4}=0$. Note that the interactions between blocking factors and treatment factors are assumed to be not existent and are not counted here. The confounding pattern of $d_{1}$ is $(4,4,0)$ which means that four 2-factor interactions and four 3-factor interactions not in the model are confounded with the effects in the model. For $d_{2}$, we assign the four treatment factors $A, B, C$, and $D$ to columns $4,2,3$, and 1 , and the blocking factor to column $5_{b}$. The interactions to be estimated should be (4)(2) and (4)(3). Since $3=(1)(2)$ and $5_{b}=(1)(4)$, the defining contrast subgroup of $d_{2}$ is given by $I=(1)(2)(3)=(1)(4)\left(5_{b}\right)=(2)(3)(4)\left(5_{b}\right)$. Hence we have $1=(2)(3), 2$ $=(1)(3), 3=(1)(2), 5_{b}=(1)(4), 5_{b}=(2)(3)(4),(2)(4)=(1)(3)(4),(3)(4)=(1)(2)(4)$, and (4) $=$ $(1)(2)(3)(4)$. Hence the confounding pattern of $d_{2}$ is $(4,3,1)$ meaning that four 2 -factor interactions, three 3-factor interactions, and one 4-factor interaction not included in the model are confounded with the effects in the model. Based on our design selection criterion, $d_{2}$ is better than $d_{1}$ because $N_{2}\left(d_{1}\right)=N_{2}\left(d_{2}\right)$ and $N_{3}\left(d_{1}\right)>N_{3}\left(d_{2}\right)$ where $N_{2}\left(d_{1}\right), N_{2}\left(d_{2}\right), N_{3}\left(d_{1}\right)$, and $N_{3}\left(d_{2}\right)$ denote the $N_{2}$ and $N_{3}$ for $d_{1}$ and $d_{2}$ respectively.

\subsection{Theoretical justification of the criterion}

Suppose that we are interested in estimating all main effects, a set of important two-factor interactions by using a blocked $2^{m-p}$ designs. Then the fitted model is given by

$$
Y=\beta_{0} I+W_{1} \lambda_{1}+\varepsilon
$$

where $Y$ denotes the vector of $n$ observations, $\beta_{0}$ is the grand mean, $I$ denotes the vector of $n$ ones, $\lambda_{1}$ is the vector of parameters containing all main effects, a set of important two-factor interactions, and blocking effects, $W_{1}$ is the corresponding design matrix, and $\varepsilon$ is the vector of uncorrelated random errors, assumed to have mean 0 and a constant variance. Since other interactions among treatment factors may not be negligible, the true model can be written as

$$
Y=\beta_{0} I+W_{1} \lambda_{1}+X_{2} \beta_{2}+X_{3} \beta_{3}+\cdots+X_{m} \beta_{m}+\varepsilon
$$

where $\beta_{2}$ is the vector of remaining two-factor interactions and $X_{2}$ is the corresponding design matrix, $\beta_{j}$ is the vector of $j$ factors interactions and $X_{j}$ is the corresponding matrix. The least square estimator $\hat{\lambda}_{1}=\left(W_{1}^{T} W_{1}\right)^{-1} W_{1}^{T} Y=n^{-1} W_{1}^{T} Y$ from the fitted model in (1) has expectation, taken under the true model in (2), of $E\left(\hat{\lambda}_{1}\right)=\lambda_{1}+P_{2} \beta_{2}+P_{3} \beta_{3}+\cdots+P_{m} \beta_{m}$, where $P_{2}=$ $n^{-1} W_{1}^{T} X_{2}$ and $P_{j}=n^{-1} W_{1}^{T} X_{j}$. So the bias of $\hat{\lambda}_{1}$ for estimating $\lambda_{1}$ is given by (Ke and Tang, 2003)

$$
\operatorname{Bias}\left(\hat{\lambda}_{1}, \lambda_{1}\right)=P_{2} \beta_{2}+P_{3} \beta_{3}+\cdots+P_{m} \beta_{m}
$$

Note $P_{j} \beta_{j}$ is the contribution of $\beta_{j}$ to the bias. Because $\beta_{j}$ is unknown, we have to work with $P_{j}$. One size measure for a matrix $P=\left(p_{i j}\right)$ is given by $\|P\|^{2} \stackrel{\text { def }}{=} \operatorname{trace}\left(P^{\mathrm{T}} P\right)=\sum_{i, j} p_{i j}^{2}$. Under the hierarchical assumption that lower order effects are more important than higher order effects, to minimize the bias of $\hat{\lambda}_{1}$ we should sequentially minimize $\left\|P_{2}\right\|^{2}, \ldots,\left\|P_{m}\right\|^{2}$. For regular $2^{m-p}$ 
designs, the entries of $P_{j}$ are 0 or 1 , and thus $\left\|P_{j}\right\|^{2}$ is simply the number of $j$-factor interactions aliased with the effects in the postulated model in (1). Now let $N_{j}=\left\|P_{j}\right\|^{2}$. Based on the above results, we can select blocked $2^{m-p}$ designs by sequentially minimizing $N_{2}, \ldots, N_{m}$ where $N_{j}$ is the number of $j$-factor interactions not in the model and aliased with the effects in the model.

\section{Searching for minimum $N$-aberration blocked $2^{m-p}$ designs}

\subsection{Search method}

In this paper, we consider blocked $2^{m-p}$ designs of 8 runs and 16 runs. For designs of 8 runs, 7 columns compose a saturated design. The choice of blocked $2^{m-p}$ designs is limited and the optimal design is easy to select according to our criterion. For 16 run, a saturated design composes 15 columns, providing many choices of blocked $2^{m-p}$ designs. The optimal 16 run design, using our criterion, is not easy to select. We use a computer program to calculate the confounding pattern for each choice of design for a given number of treatment factors, important two-factor interactions and blocking factors. Then select the one that has minimum $N_{2}$. If two designs have equal $N_{2}$, we select the design that has the minimum $N_{3}$, and so on. Through our search effort, we have found all existing minimum $N$-aberration optimal blocked $2^{m-p}$ designs of 8 runs, and optimal designs of 16 runs for the models containing up to three 2-factor interactions and one blocking factor. Let $k$ be the number of important 2-factor interactions. For $k=1$, there is only one model, as represented by Figure 1. Each point represents a factor and connected points identify 2-factor interaction in the model. For $k=2,3$, the number of models is 2 and 5 respectively, and the graphs for these models are given in Figures 2 and 3 respectively. In our search effort, we have used $\left(N_{2}, N_{3}, N_{4}\right)$ instead of the entire vector $\left(N_{2}, \ldots, N_{m}\right)$ to reduce the computing burden. Actually five-factor and higher order interactions are very small and are usually negligible in practice.

\subsection{Optimal blocked $2^{m-p}$ minimum $N$-aberration designs of 8 and 16 runs}

Table 2 presents all existing optimal designs of 8 runs for all. Tables 3-5 present the optimal designs of 16 runs for the models containing one blocking factor and one, two, and three important two-factor interactions respectively. In these tables, the entries under " $m_{t}+m_{b}$ " give the number of treatment factors plus the number of blocking factors, the entries under "model" indicate which model is under consideration, and for example, an entry of 2(a) denotes the model represented by Figure 2(a). The entries under "treatment factor" give the additional columns in addition to the independent columns (which are 1, 2, and 4 for 8-run designs and 1, 2, 4, and 8 for 16-run designs) for the main effects in the fitted model. The entries under "block factor" give the columns of the blocking factors. Column $j$ in these tables denotes the $j$-th column in the saturated design with its columns arranged in Yates order. The Yates's order of the columns of a 16-run saturated design can be written as $\left(\boldsymbol{a}_{\mathbf{1}}, \boldsymbol{a}_{\mathbf{2}}, a_{1} a_{2}, \boldsymbol{a}_{\mathbf{3}}, a_{1} a_{3}, a_{2} a_{3}, a_{1} a_{2} a_{3}, \boldsymbol{a}_{\mathbf{4}}, a_{1} a_{4}, a_{2} a_{4}\right.$, $\left.a_{1} a_{2} a_{4}, a_{3} a_{4}, a_{1} a_{3} a_{4}, a_{2} a_{3} a_{4}, a_{1} a_{2} a_{3} a_{4}\right)$ where other columns can be generated from the four independent columns $\boldsymbol{a}_{1}, \boldsymbol{a}_{2}, \boldsymbol{a}_{3}$, and $\boldsymbol{a}_{\mathbf{4}}$. The entries under "2-f interaction" show how to assign the factors involved in the important 2 -factor interactions. The last column in these tables gives $\left(N_{2}, N_{3}, N_{4}\right)$. 


\section{An illustrative example for an agricultural experiment}

The optimal blocked $2^{m-p}$ designs of 8 runs and 16 runs are listed in Tables $2-5$. When we plan to study several treatment factors and some important 2-factor interactions by using a blocked $2^{m-p}$ design of 8 runs or 16 runs, we can choose an optimal design directly from these tables to satisfy our needs.

Suppose that in an agricultural experiment, the experimenter wants to study six factors: Nitrogen, Phosphorus, Potassium, temperature, moisture, and light. She would like to use a blocked $2^{m-p}$ design of 16 runs with one blocking factor that is the subdivision of the field. In addition to the main effects of these factors, she also wants to estimate the three two-factor interactions that are between Nitrogen and Phosphorus, between Nitrogen and Potassium, and between Phosphorus and Potassium. The graph for this model is 3(e) as in Figure 3. The optimal design for this model can be found in Table 5. Now let us look at the row for $m_{t}+m_{b}=6+1$ and model 3(e) in Table 5. We see that the additional columns for treatment factors are 7 and 11 (together with columns 1,2, 4, and 8) and the column for blocking factor is 13. To complete the specification of the optimal design, we need to appropriately assign the six treatment factors to the six columns 1, 2, 4, 8, 7, and 11. The "2-f interaction" column in Table 5 indicates that we should assign Nitrogen, Phosphorus, and Potassium to columns 1, 4, and 8 and assign other treatment factors to columns 2,7 , and 11. This design has $N_{2}=3$, meaning that three 2-factor interactions not in the model are confounded with the effects in the model. This design is the minimum $N$-aberration design.

\section{Summary}

In this paper, we proposed and studied a method for efficiently selecting minimum $\mathrm{N}$-aberration blocked two-level fractional factorial designs when some of the two-factor interactions are important. We described how to construct and search for these designs and presented some results for designs of 8 and 16 runs. Optimal design tables were provided for the given number of treatment factors, blocking factors, and the important two-factor interactions. An example was used to illustrate how to apply the optimal design tables to an agricultural experiment.

\section{Acknowledgment}

This material is based upon work supported by the Research/Scholarship Support Fund of South Dakota State University.

\section{References}

Addelman, S. (1962). Symmetrical and asymmetrical fractional factorial plans. Technometrics 1, 47-58.

Bisgaard, S. (1994). Blocking Generators for small $2^{k-p}$ designs. Journal of Quality Technology 26, 288-296.

Box, G.E.P. and Hunter, J.S. (1961). The $2^{k-p}$ fractional factorial designs. Technometrics 
3, 311-351, 449-458.

Box, G.E.P., Hunter, W.G. \& Hunter, J.S. (1978). Statistics for Experimenters. Wiley.

Chen, H., and Cheng, C. S. (1999). Theory of optimal blocking of $2^{n-m}$ designs. The Annals of Statistics 27, 1948-1973.

Chen, J., Sun, D.X., and Wu, C. F. J. (1993). A catalogue of two-level and three-level fractional factorial designs with small runs. International Statistical Review 61, 131-145.

Cheng, C.S. and Tang, B. (2005). A general theory of minimum aberration and its applications. Annals of Statistics 33, 944-958.

Cheng, S.-W., Li, W.. and Ye, K. Q. (2004). Blocked non-regular two-level fractional factorial designs. Technometrics 46, 269-279.

Cheng, S.-W. and Wu, C. F. J. (2002). Choice of optimal blocking schemes in two-level and three-level designs. Technometrics 44, 269-277.

Dey A. and Suen, C.-Y. (2002). Optimal factorial plans for main effects and two-factor interactions: A projective geometric approach. The Annals of Statistics 30, 1512-1523.

Franklin, M.F. (1984). Constructing tables of minimum aberration $p^{n-m}$ designs. Technometrics 26, 225-232.

Franklin, M.F. (1985). Selecting defining contrasts and confounded effects in $p^{n-m}$ factorial experiment. Technometrics 27, 165-172.

Fries, A. and Hunter, W.G. (1980). Minimum aberration $2^{k-p}$ designs. Technometrics 22, 601-608.

Greenfield, A.A. (1976). Selection of defining contrasts in two-level experiments. Applied Statistics 25, 64-67.

Hedayat, A. S. and Pesotan (1992). Two-level fractional designs for main effects and selected two-factor interactions. Statistica Sinica 2, 453-464.

Ke, W. and Tang, B. (2003). Selecting $2^{m-p}$ designs using a minimum aberration criterion when some two-factor interactions are important. Technometrics 45, 352-360.

Ke, W., Tang, B., and Wu, H (2005). Compromise plans with clear two-factor interactions. Statistica Sinica 15, Number 3, 709-715.

Sitter, R. R., Chen, J. and Feder, M. (1997). Fractional resolution and minimum aberration in blocked $2^{n-k}$ Designs. Technometrics 39, 382-390.

Sun, D. X., Wu, C. F. J., and Chen, Y. (1997). Optimal blocking Schemes for $2^{n}$ and $2^{n-p}$ Designs. Technometrics 39, 298-307.

Tang, B and Deng, L.Y. (1999). Minimum $G_{2}$-aberration for non-regular fractional factorial designs. Annals of Statistics 27, 1914-26.

Wu, C. F. J. and Chen, Y. (1992). A graph-aided method for planning two-level experiments 
when certain interactions are important. Technometrics 34, 162-175.

Wu, C. F. J. and Hamada, M. (2000). Experiments: Planning, Analysis, Parameter Design Optimization. New York: Wiley.

Table 1. Columns of an 8-run saturated design in Yates's order

\begin{tabular}{|c|c|c|c|c|c|c|c|c|}
\hline Run & $\mathbf{1}$ & $\mathbf{2}$ & $\begin{array}{c}3 \\
3=(1)(2)\end{array}$ & $\mathbf{4}$ & $\begin{array}{c}5 \\
5=(1)(4)\end{array}$ & $\begin{array}{c}6 \\
6=(2)(4)\end{array}$ & $\begin{array}{c}7 \\
7=(1)(2)(4)\end{array}$ & Response \\
\hline 1 & $\mathbf{- 1}$ & $\mathbf{- 1}$ & +1 & $\mathbf{- 1}$ & +1 & +1 & -1 & $y_{1}$ \\
\hline 2 & $\mathbf{+ 1}$ & $\mathbf{- 1}$ & -1 & $\mathbf{- 1}$ & -1 & +1 & +1 & $y_{2}$ \\
\hline 3 & $\mathbf{- 1}$ & $\mathbf{+ 1}$ & -1 & $\mathbf{- 1}$ & +1 & -1 & +1 & $y_{3}$ \\
\hline 4 & $\mathbf{+ 1}$ & $\mathbf{+ 1}$ & +1 & $\mathbf{- 1}$ & -1 & -1 & -1 & $y_{4}$ \\
\hline 5 & $\mathbf{- 1}$ & $\mathbf{- 1}$ & +1 & $\mathbf{+ 1}$ & -1 & -1 & +1 & $y_{5}$ \\
\hline 6 & $\mathbf{+ 1}$ & $\mathbf{- 1}$ & -1 & $\mathbf{+ 1}$ & +1 & -1 & -1 & $y_{6}$ \\
\hline 7 & $-\mathbf{1}$ & $\mathbf{+ 1}$ & -1 & $+\mathbf{1}$ & -1 & +1 & -1 & $y_{7}$ \\
\hline 8 & $+\mathbf{1}$ & $+\mathbf{1}$ & +1 & $+\mathbf{1}$ & +1 & +1 & +1 & $y_{8}$ \\
\hline
\end{tabular}

Table 2. Optimal blocked designs of 8 runs for the models containing some 2-f interactions and one block factor

\begin{tabular}{ccccc}
\hline$m_{t}+m_{b}$ & treatment factor & 2-f interaction & block factor & $\left(N_{2}, N_{3}, N_{4}\right)$ \\
\hline $4+1$ & 7 & $(1,4)$ & 3 & $(3,4,0)$ \\
$5+1$ & 35 & $(2,5)$ & 6 & $(9,8,4)$ \\
$4+1$ & 3 & $(2,4)(3,4)$ & 5 & $(4,3,1)$ \\
\hline
\end{tabular}


Table 3. Optimal blocked designs of 16 runs for the model containing one 2-f interaction and one block factor

\begin{tabular}{|c|c|c|c|c|}
\hline$m_{t}+m_{b}$ & treatment factor & 2-f interaction & block factor & $\left(N_{2}, N_{3}, N_{4}\right)$ \\
\hline $5+1$ & 7 & $(1,8)$ & 11 & $(0,6,1)$ \\
\hline $6+1$ & 711 & $(1,4)$ & 13 & $(1,16,2)$ \\
\hline $7+1$ & 71113 & $(1,2)$ & 14 & $(2,37,4)$ \\
\hline $8+1$ & 7111314 & $(1,2)$ & 5 & $(7,56,16)$ \\
\hline $9+1$ & 3591415 & $(2,4)$ & 7 & $(19,64,80)$ \\
\hline $10+1$ & 35691415 & $(2,8)$ & 11 & $(31,88,160)$ \\
\hline $11+1$ & 3569101314 & $(1,6)$ & 15 & $(44,129,272)$ \\
\hline $12+1$ & 1356910131415 & $(1,6)$ & 11 & $(59,188,432)$ \\
\hline $13+1$ & 35691011131415 & $(1,6)$ & 12 & $(77,264,660)$ \\
\hline
\end{tabular}

Table 4. Optimal blocked designs of 16 runs for the models containing two 2-f interactions, as in Figure 2, and one block factor

\begin{tabular}{|c|c|c|c|c|c|}
\hline$m_{t}+m_{b}$ & model & treatment factor & 2-f interaction & block factor & $\left(N_{2}, N_{3}, N_{4}\right)$ \\
\hline $5+1$ & $2(a)$ & 15 & $(1,2)(4,8)$ & 5 & $(1,3,5)$ \\
\hline $5+1$ & 2(b) & 7 & $(1,8)(2,8)$ & 11 & $(0,6,2)$ \\
\hline $6+1$ & 2(a) & 711 & $(1,4)(2,8)$ & 13 & $(2,16,4)$ \\
\hline $6+1$ & $2(b)$ & 711 & $(1,4)(2,4)$ & 13 & $(2,16,4)$ \\
\hline $7+1$ & 2(a) & 71113 & $(1,2)(4,8)$ & 14 & $(4,35,8)$ \\
\hline $7+1$ & $2(b)$ & 71113 & $(1,2)(1,4)$ & 14 & $(4,35,8)$ \\
\hline $8+1$ & 2(a) & 7111314 & $(1,2)(4,8)$ & 5 & $(10,56,24)$ \\
\hline $8+1$ & 2(b) & 7111314 & $(1,2)(1,4)$ & 6 & $(10,56,24)$ \\
\hline $9+1$ & 2(a) & 3591415 & $(2,4)(3,8)$ & 7 & $(22,68,88)$ \\
\hline $9+1$ & $2(b)$ & 3591415 & $(2,4)(3,4)$ & 10 & $(22,68,88)$ \\
\hline $10+1$ & 2(a) & 35691415 & $(2,8)(3,14)$ & 11 & $(34,96,172)$ \\
\hline $10+1$ & 2(b) & 35691415 & $(2,8)(3,8)$ & 12 & $(34,96,172)$ \\
\hline $11+1$ & $2(a)$ & 3569101314 & $(1,10)(2,5)$ & 15 & $(48,141,288)$ \\
\hline $11+1$ & 2(b) & 3569101314 & $(1,13)(2,13)$ & 7 & $(48,141,288)$ \\
\hline $12+1$ & 2(a) & 35679101112 & $(1,12)(4,10)$ & 15 & $(64,203,457)$ \\
\hline $12+1$ & 2(b) & 35679101112 & $(1,12)(2,12)$ & 15 & $(64,203,457)$ \\
\hline
\end{tabular}


Table 5. Optimal blocked designs of 16 runs for the models containing three 2-f interactions, as in Figure 3, and one block factor (A row having entries "-" indicates the situation where the specified model does not exist for the given number of factors.)

\begin{tabular}{|c|c|c|c|c|c|}
\hline$m_{t}+m_{b}$ & model & treatment factor & 2-f interaction & block factor & $\left(N_{2}, N_{3}, N_{4}\right)$ \\
\hline $5+1$ & $3(\mathrm{a})$ & - & - & - & - \\
\hline $5+1$ & $3(b)$ & 15 & $(1,2)(4,8)(4,15)$ & 5 & $(1,4,5)$ \\
\hline $5+1$ & $3(\mathrm{c})$ & 7 & $(1,8)(2,8)(4,8)$ & 11 & $(0,6,3)$ \\
\hline $5+1$ & $3(d)$ & 15 & $(1,4)(4,8)(8,2)$ & 3 & $(1,4,5)$ \\
\hline $5+1$ & $3(\mathrm{e})$ & 15 & $(1,2)(1,4)(2,4)$ & 7 & $(1,4,5)$ \\
\hline $6+1$ & 3(a) & 711 & $(1,4)(2,8)(7,11)$ & 13 & $(3,16,6)$ \\
\hline $6+1$ & $3(\mathrm{~b})$ & 711 & $(1,4)(2,8)(7,8)$ & 13 & $(3,16,6)$ \\
\hline $6+1$ & $3(\mathrm{c})$ & 711 & $(1,4)(2,4)(4,8)$ & 13 & $(3,16,6)$ \\
\hline $6+1$ & $3(d)$ & 711 & $(1,4)(4,8)(8,2)$ & 13 & $(3,16,6)$ \\
\hline $6+1$ & $3(\mathrm{e})$ & 711 & $(1,4)(1,8)(4,8)$ & 13 & $(3,16,6)$ \\
\hline $7+1$ & $3(a)$ & 71113 & $(1,4)(2,8)(7,11)$ & 14 & $(6,35,12)$ \\
\hline $7+1$ & 3(b) & 71113 & $(1,2)(4,8)(7,8)$ & 14 & $(6,35,12)$ \\
\hline $7+1$ & $3(\mathrm{c})$ & 71113 & $(1,2)(1,4)(1,7)$ & 14 & $(6,35,12)$ \\
\hline $7+1$ & $3(d)$ & 71113 & $(1,4)(4,8)(8,2)$ & 14 & $(6,35,12)$ \\
\hline $7+1$ & $3(\mathrm{e})$ & 71113 & $(1,2)(1,4)(2,4)$ & 14 & $(6,35,12)$ \\
\hline $8+1$ & $3(a)$ & 7111314 & $(1,4)(2,8)(7,11)$ & 3 & $(13,56,32)$ \\
\hline $8+1$ & 3(b) & 7111314 & $(1,2)(4,8)(7,8)$ & 5 & $(13,56,32)$ \\
\hline $8+1$ & $3(\mathrm{c})$ & 7111314 & $(1,2)(1,4)(1,7)$ & 9 & $(13,56,32)$ \\
\hline $8+1$ & $3(d)$ & 7111314 & $(1,4)(4,8)(8,2)$ & 3 & $(13,56,32)$ \\
\hline $8+1$ & $3(\mathrm{e})$ & 7111314 & $(1,2)(1,4)(2,4)$ & 9 & $(13,56,32)$ \\
\hline $9+1$ & $3(\mathrm{a})$ & 3591415 & $(2,4)(3,8)(5,9)$ & 7 & $(25,72,96)$ \\
\hline $9+1$ & $3(b)$ & 3591415 & $(2,4)(3,8)(5,8)$ & 7 & $(25,72,96)$ \\
\hline $9+1$ & $3(\mathrm{c})$ & 3591415 & $(2,4)(3,4)(4,8)$ & 10 & $(25,72,96)$ \\
\hline $9+1$ & $3(d)$ & 3591415 & $(2,4)(4,8)(8,3)$ & 7 & $(25,72,96)$ \\
\hline $9+1$ & $3(e)$ & 3591415 & $(2,4)(2,8)(4,8)$ & 7 & $(25,72,96)$ \\
\hline $10+1$ & $3(\mathrm{a})$ & 35691415 & $(2,9)(3,14)(4,8)$ & 10 & $(37,104,184)$ \\
\hline $10+1$ & $3(b)$ & 35691415 & $(4,8)(2,9)(3,9)$ & 13 & $(37,104,184)$ \\
\hline $10+1$ & $3(\mathrm{c})$ & 35691415 & $(2,8)(3,8)(4,8)$ & 13 & $(37,104,184)$ \\
\hline $10+1$ & $3(d)$ & 35691415 & $(2,14)(14,3)(3,8)$ & 10 & $(37,104,184)$ \\
\hline $10+1$ & $3(\mathrm{e})$ & 35691415 & $(2,5)(2,8)(5,8)$ & 11 & $(38,104,180)$ \\
\hline $11+1$ & $3(\mathrm{a})$ & 356791011 & $(4,9)(5,10)(6,8)$ & 12 & $(52,152,304)$ \\
\hline $11+1$ & $3(\mathrm{~b})$ & 356791011 & $(6,8)(4,9)(5,9)$ & 15 & $(52,152,304)$ \\
\hline $11+1$ & $3(\mathrm{c})$ & 356791011 & $(4,8)(5,8)(6,8)$ & 15 & $(52,152,304)$ \\
\hline $11+1$ & $3(d)$ & 356791011 & $(4,10)(10,5)(5,8)$ & 12 & $(52,152,304)$ \\
\hline $11+1$ & $3(\mathrm{e})$ & 3569101314 & $(1,6)(1,10)(6,10)$ & 15 & $(52,153,304)$ \\
\hline
\end{tabular}


Figure 1. Graph for model with one 2-factor interaction.

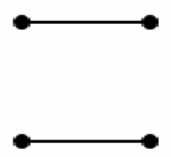

(a)

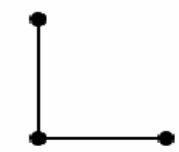

(b)

Figure 2. Graphs for models with two 2-factor interactions.

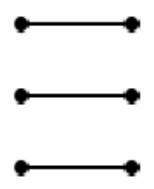

(a)

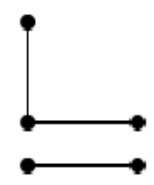

(b)

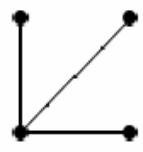

(c)

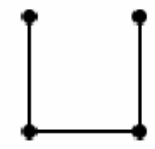

(d)

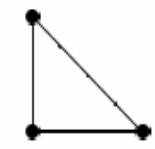

(e)

Figure 3. Graphs for models with three 2-factor interactions 